\title{
SUPRARRENALECTOMÍA LAPAROSCÓPICA RETROPERITONEAL COMO OPCIÓN MINIMAMENTE INVASIVA EN EL TRATAMIENTO DE TUMORES ADRENALES.
}

\author{
Marcos Tobias-Machado, Freddy Rincón Ríos, Marco Tulio Lasmar, Rodrigo Tristão, Pedro \\ Hermínio Forseto Jr, Roberto Vaz Juliano y Eric Roger Wroclawski.
}

Disciplina de Urología. Facultad de Medicina de ABC. Santo André. Sâo Paulo. Brasil.

\begin{abstract}
Resumen.- OBJETIVOS: La extirpación de la glándula suprarrenal por vía laparoscópica es el tratamiento estándar de oro para el tratamiento de los tumores de la adrenal menores de $6 \mathrm{~cm}$. La mayoría de los centros latinoamericanos adopta la técnica transperitoneal como vía de acceso. El objetivo del presente estudio prospectivo fue demostrar los resultados intra y postoperatorios de los pacientes sometidos a cirugía laparoscópica de la adrenal por un único cirujano a través de acceso retroperitoneal lateral.
\end{abstract}

MÉTODOS: Entre enero de 2000 y octubre de 2004, 35 pacientes (16 hombres y 19 mujeres) con lesiones adrenales, incluyendo 4 casos de sindrome de Cushing, 4 de sindrome de Conn, 1 neurogangliomas, 4 feocromocitomas, 17 adenomas no funcionantes, 1 tumor virilizante y 4 casos que se trataban de nódulo postratamiento de neoplasia primaria no adrenal, fueron sometidos prospectivamente a adrenalectomía retroperitoneoscópica por un único cirujano. Describimos el tiempo quirúrgico, la pérdida sanguínea, el tiempo para alimentación oral, el uso de analgésicos, las complicaciones quirúrgicas, y tasa de conversión, el período de hospitalización y el período de retorno a las actividades habituales, comparando los resultados con otras series de la literatura.

RESULTADOS: Todos los procedimientos fueron realizados con éxito. El tiempo quirúrgico promedio fue de 135 minutos, sangrado operatorio promedio fue de $235 \mathrm{ml}$, el período promedio para alimentación oral fue de 12 horas, ningún paciente necesitó de analgesia después del $2^{\circ}$ día postoperatorio, el período promedio de hospitalización fue de 2 días y el tiempo promedio para retornar a las actividades habituales fue de 3 semanas. Como complicaciones observamos un caso con hipercapnea intraoperatoria y un caso que evolucionó con neumonía en el postoperatorio. En ningún caso hubo necesidad de conversión para cirugía abierta.

CONCLUSIONES: La adrenalectomía endoscópica retroperitoneal puede ser realizada de manera segura y eficiente, con bajo índice de complicaciones, independiente de la etiología de la lesión. El acceso retroperitoneoscópico lateral es una excelente opción para el tratamiento minimamente invasivo de las glándulas suprarrenales.

Palabras clave: Adrenalectomía laparoscópica. Suprarrenalectomía laparoscópica. Acceso retroperitoneal. Cirugía de las glándulas adrenales. Laparoscopía. Tumores adrenales.

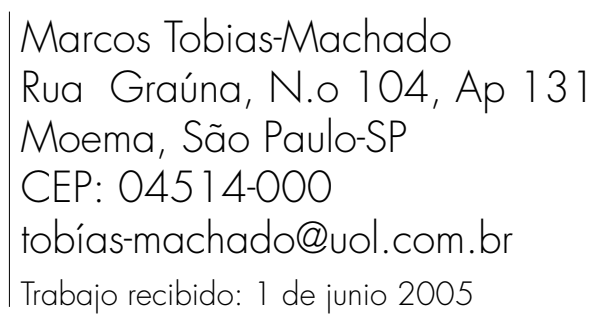


Summary.- OBJECTIVES: The excision of the adrenal gland by laparoscopic approach is the gold standard for the treatment of adrenal tumors smaller than $6 \mathrm{~cm}$. Most Brazilian centres favour the transperitoneal approach. The objective of the present prospective study was to show the intraoperative and postoperative results of patients undergoing adrenal laparoscopic surgery through a lateral retroperitoneal approach performed by an individual surgeon.

METHODS: Between January 2000 and October 2004, 35 patients (16 males and 19 females) with adrenal lesions, including 4 Cushing's syndrome, 4 Conn's syndrome, 1 neuroganglioma, 4 pheochromocytoma, 17 nonfunctioning adenomas, 1 virilizing tumor, and 4 cases of adrenal nodule after treatment of a non-adrenal primary neoplasia, prospectively underwent retroperitoneoscopic adenomectomy by one surgeon. We describe surgical times, estimated blood losses, time to oral intake, analgesic requirements, surgical complications and conversion rates, hospital stay and time to return to usual activities, comparing our results with other series in the literature.

RESULTS: All procedures were successfully completed. Mean surgical time was 135 minutes, mean estimated blood loss $235 \mathrm{ml}$, mean time to oral intake 12 hours, no patient required analgesics affer the second postoperative day, mean hospital stay was 2 days, and mean time to return to usual life activities 3 weeks. There were two complications, one case of intraoperative hypercapnia and one case with postoperative pneumonia. Conversion to open surgery was not required in any case.

CONCLUSIONS: Retroperitoneoscopic adrenalectomy may be performed effectively and safely, with a low complication rate, independently of the etiology of the lesion. The lateral retroperitoneoscopic access is an excellent option for the minimally invasive treatment of the adrenal glands.

Keywords: Laparoscopic adrenalectomy. Retroperitoneoscopy. Adrenal gland surgery. Laparoscopy. Adrenal tumors.

\section{INTRODUCCIÓN}

La adrenalectomía laparoscópica fue inicialmente descrita por Gagner y cols.(1) en 1992 y desde entonces ha sido ampliamente utilizada. Constituye actualmente el estándar de oro para el tratamiento de lesiones adrenales menores de 6 centímetros. Varios trabajos demuestran su efectividad, proporcionando menor trauma quirúrgico y ventajas postoperatorias inequívocas en relación a la cirugía convencional (2-3).

A pesar de ser bastante difundida actualmente, no hay consenso en la literatura en relación al mejor acceso minimamente invasivo. En Centro América, las cirugías laparoscópicas de las adrenales son realizadas casi exclusivamente por acceso transperitoneal (4).

El objetivo del presente trabajo fue describir los resultados quirúrgicos de un estudio prospectivo utilizando acceso retroperitoneoscópico lateral en el tratamiento de los nódulos adrenales. Los hallazgos fueron discutidos considerando otros estudios pertinentes en la literatura.

\section{PACIENTES Y MÉTODOS}

\section{Pacientes}

Durante el período de enero de 2000 y octubre de 2004, 35 pacientes 116 hombres y 19 mujeres) con tumores adrenales fueron sometidos a adrenalectomía retroperitoneoscópica por un único cirujano y seguidos prospectivamente.

Todos los pacientes pasaron por evaluación funcional y de laboratorio con endocrinología, así como también por exámenes radiológicos para evaluación de la localización y tamaño de la lesión - Tomografía Computarizada (TC) en todos los casos y Resonancia Nuclear Magnética (RNM) en los casos de tumor mayor de $4 \mathrm{~cm}$ o con signos sugestivos de malignidad con la TC (lesiones heterogéneas o con desimpregnación lenta del contraste).

Los diagnósticos clínicos preoperatorios fueron síndrome de Cushing [ 4 casos], síndrome de Conn [ 4], feocromocitomas [ 4], neurogangliomas [ 1 ], adenomas no funcionantes - todos mayores que 3 $\mathrm{cm}-[17$ ], tumor virilizante [ 1 ], y 4 casos representaban nódulos postratamiento de neoplasia primaria no adrenal (anatomo-patológico final reveló 2 casos de enfermedad metastásica de neoplasia primaria de mama y de pulmón y otros 2 casos de adenomas). Veinte neoplasias eran del lado derecho, mientras 
que 15 eran del lado izquierdo. Todos los pacientes fueron sometidos a anestesia general, siendo la analgesia postoperatoria realizada con analgésicos menores y/o anti-inflamatorios no esteroideos. No hubo necesidad de opiáceo en ningún caso del protocolo. Los parámetros evaluados fueron: indicación clínica, tiempo quirúrgico, pérdida sanguínea intra-operatoria, complicaciones intra y postoperatorias, analgesia postoperatoria, tiempo para alimentación oral, período de internación y período para el retorno a las actividades habituales. El seguimiento y evaluación de los pacientes fueron realizados ambulatoriamente de forma estándar en el $7^{\circ}, 30^{\circ}$ y $60^{\circ}$ días del alta hospitalaria y un año después de esta fecha.

\section{Técnica quirúrgica}

Los pacientes fueron colocados en decúbito lateral e hiper-extensión, siendo el acceso obtenido a través de 4 trocares, con 3 subcostales y 1 encima de la cresta ilíaca. (Figura 1) (5).

El espacio de trabajo fue creado entre la musculatura posterior y el riñón a través de disección digital y empleando balón dilatador de retroperitoneo.

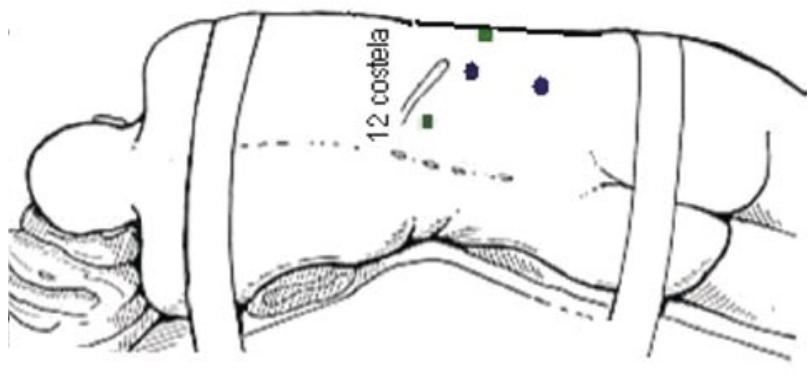

Trocar de $10 \mathrm{~mm}$

Trocar de $5 \mathrm{~mm}$

FIGURA 1. El acceso inicial al retroperitoneo es obtenido a través de incisión transversa de $1.5 \mathrm{~cm}$ debajo de la extremidad de la $12^{\underline{a}}$ costilla y después de ser creado el espacio en el retroperitoneo es introducido el trocar de $10 \mathrm{~mm}$ y óptica de cero grado. El segundo trocar $(10 \mathrm{~mm}$ ) es posicionado $2 \mathrm{~cm}$ encima de la cresta ilíaca, en el piso del triángulo lumbar inferior. Enseguida la óptica es transferida para este portal. El tercer trocar $(5 \mathrm{~mm})$ es ubicado $1.5 \mathrm{~cm}$ inferior y lateral al ángulo formado entre el borde inferior de la $12^{a}$ costilla y la musculatura paravertebral. Este portal y el portal de acceso son utilizados por el cirujano.

Un cuarto portal $(5 \mathrm{~mm})$ es manipulado por el asistente para afastamiento de estructuras adyacentes y es introducido debajo de la extremidad de la $1^{1}{ }^{a}$ costilla.
El menor espacio de trabajo y la coloración más rojiza de la imagen del campo operatorio exigen una mayor capacidad de adaptación de movimientos entre los componentes del equipo quirúrgico.

Debido a disponibilidad en la institución, se utilizó la cámara con óptica de cero grado, manteniendo la imagen del psoas en el piso del vídeo. El cirujano, el cual se posiciona posteriormente al paciente, opera prácticamente todo el tempo con una pinza para hemostasia/disección en la mano dominante y el aspirador en la otra. El asistente se posiciona contra lateralmente, utilizando una pinza romba o un afastador de hígado para rechazar el riñón o el peritoneo, facilitando la disección al cirujano.

La fascia de Gerota es abierta en el polo superior del riñón. La técnica utilizada fue la estandarizada por Suzuki y cols., con disección en bloque a través de los reparos anatómicos para posterior identificación de la glándula (6). La disección periférica de la adrenal fue obtenida de manera romba y el control de los pequeños vasos se realizó preferentemente con ayuda del bisturí ultrasónico, el cual produce una menor cantidad de humo, o bisturí monopolar. Las venas adrenales de mayor calibre fueron controladas con clips de polímero (hemo-lock) o clipes metálicos y seccionados al final del procedimiento. Para remoción de la pieza, la adrenal es colocada en una bolsa improvisada en el retroperitoneo y retirada a través del primer trocar o de incisión ampliada de uno de los trocares de $10 \mathrm{~mm}$ cuando la pieza era de mayores dimensiones.

\section{RESULTADOS}

El tamaño promedio de las lesiones fue de 5 $\mathrm{cm}(2$ a $10 \mathrm{~cm})$, siendo 20 lesiones del lado derecho y 15 del lado izquierdo. Todos los procedimientos fueron completados con éxito. El tiempo quirúrgico promedio fue de $135 \mathrm{~min}$ (70-170) y la pérdida sanguínea intra-operatoria fue en promedio de $235 \mathrm{ml}$ (120-530). Ninguno de los pacientes necesitó de transfusión sanguínea. La analgesia fue requerida en promedio hasta 44 horas (24-96 horas) de postoperatorio. El tiempo para realimentación oral fue en promedio de 12 horas (8-24 horas).

No hubo complicaciones graves. En un caso ocurrió hipercarpnea en el intra-operatorio controlada por el anestesiólogo a través de asistencia ventilatoria adecuada. Un niño de 1,5 años con tumor virilizante presentó neumonía en el postoperatorio, permaneciendo internado durante 14 días para antibioticoterapia. 
El promedio del período de internación y del período para retorno a las actividades habituales fueron de 2 días (1-14 días) y 3 semanas (2-6 semanas), respectivamente (Tabla I).

\section{DISCUSIÓN}

La adrenalectomía laparoscópica es una técnica segura y eficaz para remoción de lesiones adrenales sólidas hasta $6 \mathrm{~cm}$ para diversas etiologías $(2-4,6)$. Si características radiológicas del tumor son fuertemente sugestivas de malignidad, la experiencia y habilidad del cirujano deben ser consideradas en la decisión del acceso a ser utilizado. Lesiones metastásicas aisladas, mas comunmente de pulmón, mama, riñón o piel, pueden también ser removidas por vía laparoscópica. La única contraindicación específica ocurre en los casos de carcinoma adrenocortical con invasión local o trombo venoso (7).

En conclusión el volumen tumoral no representa una contraindicación, no existe consenso en relación al tamaño máximo de la lesión en que el procedimiento laparoscópico pueda ser utilizado con

TABLA I. RESULTADO DE LOS PARÁMETROS ESTUDIADOS EN 35 PACIENTES SOMETIDOS A CIRUGÍA RETROPERITONEOSCÓPICA DE LAS ADRENALES.

\begin{tabular}{|c|c|}
\hline & RETROPERITONEAL \\
\hline № PACIENTES & 35 \\
\hline SEXO M:F & $16: 19$ \\
\hline EDAD(años) & $55(1,5-65)$ \\
\hline LATERALIDAD & $20 \mathrm{D} / 15 \mathrm{I}$ \\
\hline TAMANOO(cm) & $5(2-10)$ \\
\hline SANGRADO(ml) & 235 \\
\hline ANALGESIA (h) & 44 \\
\hline PROMEDIO TIEMPO & $135(70-170)$ \\
QUIRURGICO(min) & \\
\hline REALIMENTACIÓN & $12(8-24)$ \\
\hline DIAS HOSPITALIZACION & $2(1-14)$ \\
\hline RECUPERACIÓN(semanas) & 3 \\
\hline
\end{tabular}

* $p>0,05$ para todos los parámetros /diferencia sin significancia estatístical seguridad (7-12). Gagner refiere exéresis laparoscópica de lesión adrenal de $14 \mathrm{~cm}$ (11). Con respecto al acceso, algunos cirujanos creen y apoyan que masas adrenales midiendo más de $8 \mathrm{~cm}$ representa una contraindicación relativa para el acceso retroperitoneal (12). Suzuki y cols. recomiendan el acceso laparoscópico retroperitoneal para tumores menores de $5 \mathrm{~cm}(6)$. No creemos que el tamaño sea una contraindicación absoluta, ya que hemos operado casos hasta de $10 \mathrm{~cm}$. Nosotros opinamos que, lesiones mayores de $8 \mathrm{~cm}$ pueden ser abordadas con más seguridad a través de acceso transperitoneal.

En la literatura los reportes entran en conflicto en relación al acceso que determina menor tiempo quirúrgico y menor pérdida sanguínea intra-operatoria. Bonjer y cols. observaron una disminución del tiempo quirúrgico y de pérdida sanguínea intra-operatoria utilizando el acceso retroperitoneal en relación al transperitoneal (12). Por otro lado, Suzuki y cols. obtuvieron resultados similares en los dos grupos en lo referente a estos parámetros (6).

Con respecto a la lateralidad, algunos autores han propuesto un abordaje selectivo. Debido al acceso directo a la glándula, sin la necesidad de amplia movilización del colon, bazo y páncreas, el acceso retroperitoneoscópico puede ser ventajoso cuando se aborda el lado izquierdo. Miyake y cols. observaron una reducción en el tiempo quirúrgico de 528 a 267 minutos en los casos de adrenalectomía izquierda, cuando compararon el abordaje transperitoneal y retroperitoneal. Estos autores indican preferencialmente el acceso retroperitoneal para adrenalectomía izquierda, considerando los dos accesos posibles para el lado derecho (8).

La mayor parte de los trabajos encontrados en la literatura no demuestran ventajas específicas en los parámetros de recuperación postoperatoria al compararse con los diferentes accesos para cirugía laparoscópica de la adrenal. Miyake y cols. no encontraron diferencias significativas en relación a los parámetros de tiempo de convalecencia en los grupos analizados (8). Sung y cols. no encontraron diferencias significativas en el tiempo quirúrgico, pérdida sanguínea, necesidad de analgesia y tiempo de permanencia hospitalaria en los dos grupos (9). Algunos estudios sugieren ventajas en escoger el acceso de acuerdo con el diagnóstico clínico de los nódulos funcionantes.

Debido al riesgo de liberación de catecolaminas durante la cirugía, el tratamiento del feocromocitoma es considerado un desafío. La cirugía laparoscópica para feocromocitoma permite una manipulación mas delicada, siendo no solamente via- 
ble desde el punto de vista técnico, sino que también es más segura en cuanto a los parámetros hemodinámicos (13-16). La gran mayoría de los cirujanos prefiere el acceso transperitoneal, pues permite un abordaje precoz de la vena suprarrenal, reduciendo de esta manera el riesgo de liberación de catecolaminas durante la cirugía $(13,14)$. Sin embargo, algunos trabajos relatan el empleo del acceso retroperitoneal con éxito $(12,16)$. Salomón y cols., demostrando la factibilidad de control precoz de la vena suprarrenal principal, relataron 21 casos de adrenalectomía retroperitoneoscópica, no observando crisis hipertensivas paroxísticas o arritmias cardíacas (16). En el presente estudio fueron realizadas 4 adrenalectomías retroperitoneoscópicas para feocromocitoma, no ocurriendo complicaciones intra-operatorias significativas.

Con relación al síndrome de Cushing y en pacientes muy obesos, debido a la gran cantidad de tejido graso en el retroperitoneo y dificultad de definir los parámetros anatómicos, algunos autores relatan su preferencia por el acceso transperitoneal (17). No obstante, Fernández-Cruz y cols. no observaron diferencias en los dos accesos, en relación a los parámetros evaluados, en los pacientes con síndrome de Cushing (18).

La mayor parte de los trabajos muestran que la morbilidad de la cirugía laparoscópica de las adrenales es baja, siendo la tasa de conversión para cirugía abierta estimada de 0 a $17 \%$ de los casos $(4,5,19)$. En nuestra casuística no hubo ninguna conversión o de lesión visceral.

A pesar de no haber consenso entre los diversos autores, un estudio comparativo entre los diferentes accesos laparoscópicos de la adrenal mostró que la morbilidad quirúrgica fue menor con el acceso retroperitoneal en comparación con el transperitoneal $(3 \% \times 9 \%)(19)$. Las principales causas de morbilidad de la cirugía laparoscópica están relacionadas a sangrado incontrolable debido a lesión vascular, dificultad de disección en los casos de intensa adherencia a los tejidos adyacentes y lesiones viscerales. Específicamente con relación al acceso retroperitoneal, algunos trabajos muestran que parece haber una mayor predisposición a lesión intraoperatoria del páncreas. En una serie de 59 adrenalectomías retroperitoneales, fueron observados 2 casos de lesión pancreática en el intra-operatorio (19). Especial cuidado debe ser tomado cuando se realiza la adrenalectomía retroperitoneoscópica izquierda para la correcta individualización de los límites del páncreas, así evitando cuadros de pancreatitis en el postoperatorio. En caso de fístula, el acceso extraperitoneal puede tener la ventaja de evitar el contacto del jugo pancreático con la cavidad peritoneal.
Debido a la ausencia de abertura de la cavidad peritoneal y sin la manipulación de las vísceras intestinales, el acceso retroperitoneal puede presentar algunas ventajas relativas a realimentación oral más precoz. Suzuki y cols., en 41 adrenalectomías transperitoneales, encontraron 2 casos de íleo paralítico prolongado en el postoperatorio, mientras que no hubo ningún caso en 36 adrenalectomías retroperitoneales (6).

Relatos iniciales sugieren que la absorción de $\mathrm{CO}_{2}$ sería mayor con el acceso retroperitoneal, sin embargo, algunos trabajos más recientes para adrenalectomía laparoscópica no demuestran diferencias en la presión final de $\mathrm{CO}_{2}$ en los dos accesos (20). Un único evento clínico que ocurrió en este trabajo no permite una conclusión definitiva sobre el asunto. Nuestra experiencia muestra que si el control ventilatorio y de hidratación en el intra-operatorio son rigurosos, los cuadros de hipercapnea intra-operatoria serán adecuadamente controlados, sin repercusión clínica para los pacientes.

\section{CONCLUSIONES}

La adrenalectomía endoscópica retroperitoneal puede ser realizada de manera segura y eficiente, con bajo índice de complicaciones, independiente de la etiología de la lesión.

El acceso retroperitoneoscópico lateral es una excelente opción para el tratamiento minimamente invasivo de las glándulas suprarrenales.

En nuestra opinión, la elección de acceso laparoscópico depende de las peculiaridades de cada caso y de la experiencia personal del cirujano.

\section{BIBLIOGRAFÍA y LECTURAS RECOMENDADAS (*lectura de interés $y^{* *}$ lectura fundamental)}

**1. GAGNER, M.; LACROIX, A.; BOLTE, E.: "Laparoscopic adrenalectomy in Cushing's syndrome and pheocromocytoma". New Engl. J. Med., 327: 1003, 1992.

2. IMAI, T.; KIKUMORI, T.; OHIWA, M. y cols.: "A case-controlled study of laparoscopic compared with open lateral adrenalectomy". Am. J. Surg., 178: 50, 1999.

*3. KORMAN, J.E.; HO, T.; PHILIPS, E.H.: "Comparison of laparoscopic and open adrenalectomy". Am. Surg., 63: 908, 1997. 
4. CASTILHO, L.N.; MITRE, A.I.; ARAP, S.: "Laparoscopic adrenal surgery in a Brazilian center". J. Endourol., 17: 11, 2003

*5. TOBIAS-MACHADO, M.; LASMAR, M.T.; RINCÓN, F. y cols.: "Seis años de experiencia en cirugía laparoscópica lumbar extraperitoneal: indicaciones, complicaciones y resultados en un centro latino-americano de referencia". Arch. Esp. Urol., Aceptado para publicación 2005.

**6. SUZUKI, K.; KAGEYAMA, S.; HIRANO, Y. y cols.: "Comparison of 3 surgical approaches to laparoscopic adrenalectomy: a nonrandomized, background matched analysis". J. Urol., 166: 437, 2001.

*7. GILL,I.S.: "The case for laparoscopic adrenalectomy". J Urol. 166: 429, 2001.

8. MIYAKE, O.; YOSHIMURA, K.; YOSHIOKA, T.: "Laparoscopic adrenalectomy. Comparison of the transperitoneal and retroperitoneal approach". Eur. Urol., 33: 303, 1998.

9. SUNG, G.T.; GILL, I.S.; HOBART, M.: "Laparoscopic adrenalectomy: Prospective, randomized comparison of transperitoneal vs retroperitoneal approaches". J. Urol., 161: 21, 1999.

10. WINFIELD, H.N.; HAMILTON, B.D.; BRAVO, E.L.: "Technique of laparoscopic adrenalectomy". Urol. Clin. North Am., 24: 59, 1997.

11. GAGNER, M.; POMP, A.; HENIFORD, B.T. y cols.: "Laparoscopic adrenalectomy; lessons learned from 100 consecutive procedure". Ann. Surg., 226: 238, 1997.

**12. BONJER, H.J.; LANGE, J.F.; KAZERMIER, G. y cols : "Comparison of three techniques for adrenalectomy”. Br. J. Surg., 84: 679, 1997.
*13. FERNANDEZ- CRUZ, L.; TAURA, P.; SAENZ, A. y cols.: "Laparoscopic approach to pheocromocytoma: hemodynamic changes and catecholamine secretion". World J. Surg., 20: 762, 1996. MO-

14. BIUS, E.; NIES, C.; ROTHMUND, M.: "Surgical treatment of pheocromocytomas. Laparoscopic or conventional?". Surg. Endosc., 13: 35, 1999.

15. JANETSCHEK, G.; FINKENSTEDT, G.; GRASSER, R. y cols: "Laparoscopic surgery for pheocromocytoma: adrenalectomy, partial resection, excision of paragangliomas". J. Urol., 160: 330, 1998.

*16. SALOMON, L.; RABII, R.; SOULIE, M. y cols.: "Experience with retroperitoneal laparoscopic adrenalectomy for pheocromocytoma". J. Urol., 165: 1871, 2001.

17. TAKEDA, M.: "Laparoscopic adrenalectomy: transperitoneal vs retroperitoneal approaches". Biomed \& Pharmacother, 54: 207, 2000.

*18. FERNANDEZ - CRUZ, L.; SAENZ, A.; BENARROCH, G. y cols.: "Laparoscopic unilateral and bilateral adrenalectomy for Cushing's syndrome. Transperitoneal \& retroperitoneal approaches". Ann. Surg., 224: 734, 1996.

**19. TERACHI, T.; YOSHIDA, O.; MATSUDA, T. y cols.: "Complications of laparoscopic and retroperitoneoscopic adrenalectomies in 370 cases in Japan: a multi institutional study". Biomed \& Pharmacother, 54: 211, 2000.

20. NG, C.S.; GILL, I.S.; SUNG, G.T. y cols.: "Retroperitoneoscopic surgery is not associated with increased carbon dioxide absorption". J. Urol., 162: $1268,1999$. 